\title{
Proliferation of Rindera graeca transgenic roots in oscillatory rocked disposable bioreactor
}

$\underline{\text { Kamil Wierzchowski }}^{1}$, Mateusz Kawka ${ }^{2}$, Katarzyna Sykłowska-Baranek ${ }^{2}$, Maciej Pilarek ${ }^{1}$

1. Faculty of Chemical and Process Engineering, Warsaw University of Technology, POLAND, Warsaw, Waryńskiego 1, E-mail: kamil.wierzchowski.dokt@pw.edu.pl (KW), maciej.pilarek@pw.edu.pl (MP)

2. Faculty of Pharmacy, Medical University of Warsaw, POLAND, Warsaw, Banacha 1, E-mail: mateusz.kawka@wum.edu.pl (MK), katarzyna.syklowska-baranek@wum.edu.pl (KS-B)

Abstract - Plant biomass is a commonly known source of pharmaceutically valuable compounds. The study aimed to investigate the influence of wave-type agitation conditions on the proliferation of Ringera graeca hairy roots in the disposable bioreactor. Transgenic roots morphology, fresh biomass increases, and yield of extracellularly secreted bioactive compounds have been recognized.

Keywords - disposable (single-use) bioreactor, wave-assisted agitation, transgenic (hairy) roots, aeration, bioprocess intensification

\section{Introduction}

Plant biomass is a commonly known source of pharmaceutically valuable compounds exhibiting a range of biological activities [1]. In vitro cultures of plant biomass are mainly performed as small scale bioprocesses, e.g., in Erlenmeyer flasks, in which aeration of culture medium is far insufficient. A low level of oxygen dissolved in culture medium significantly limits biomass proliferation and might strongly impact on metabolic pathways [2]. Disposable (single-use) bioreactors are recognized as suitable systems for increasing and controlling level of aeration in cultures of isolated plant cells, tissues, or cultures. In such approaches, agitation is achieved by continuous horizontal oscillation movement of the whole disposable container fixed in a rocker unit. Under gentle conditions of continuous rocking, the interfacial area formed between the gas and culture medium is renewed by waving and therefore the bubble-free surface aeration of the liquid phase is successfully accomplished. Simultaneously, the generated waves induce agitation of large volumes of culture medium and facilitate the dispersion of medium components, enhancing the homogeneity of the culture microenvironment [3]. The aim of the study was to investigate the proliferation of Ringera graeca transgenic roots in the disposable bioreactor. The influence of basic operational parameters defining wave-assisted agitation, i.e., angle $(\alpha)$ and frequency $(\omega)$ of oscillations, on transgenic roots morphology, fresh biomass increase $(F B)$, and yield of extracellularly secreted secondary metabolites $\left(C_{\mathrm{NQ}}\right)$, has been recognized.

\section{Materials and methods}

ReadyToProcess WAVE 25 bioreactor system (WAVE 25) equipped with $2 \mathrm{dm}^{3}$ disposable bag-like container (Cellbag ${ }^{\mathrm{TM}} 2 \mathrm{~L}$ ), has been applied for bioprocessing of Rindera graeca hairy roots at the following ranges of the operational parameters: $\alpha=2-10^{\circ}$ and $\omega=10-40 \mathrm{~min}^{-1}$. Hairy roots of $R$. graeca were maintained for 28 days at $25{ }^{\circ} \mathrm{C}$, with harvesting samples of culture medium twice a week. Lyophilized and micronized hairy roots, as well as samples of culture medium, were extracted with organic solvent and the level of naphthoquinone-analogues secondary metabolites has been chromatographically analyzed. 


\section{Results}

The values of $F B$ and $C_{\mathrm{NQ}}$ noted for $R$. graeca hairy roots cultured in WAVE 25 at various conditions of continuously rocking resulted in wave-type agitation has been shown in Table 1 .

Table 1

The values of $F B$ and $C_{\mathrm{NQ}}$ noted for cultures of $R$. graeca hairy roots in the WAVE 25 system

\begin{tabular}{|c|c|c|c|}
\hline \multicolumn{2}{|c|}{ conditions of wave-type agitation } & \multirow{2}{*}{$\begin{array}{c}F B \\
{[-]}\end{array}$} & \multirow{2}{*}{$\begin{array}{c}C_{\mathrm{NQ}} \\
{[\mu \mathrm{g}]}\end{array}$} \\
\hline$\alpha\left[^{0}\right]$ & $\omega\left[\min ^{-1}\right]$ & & \\
\hline \multirow{4}{*}{6} & 10 & 1.85 & 420.0 \\
\hline & 20 & 5.38 & 134.8 \\
\hline & 30 & 1.50 & 75.12 \\
\hline & 40 & \multicolumn{2}{|c|}{ disintegration of biomass } \\
\hline 2 & \multirow{5}{*}{20} & 8.14 & 384.8 \\
\hline 4 & & 6.22 & 228.2 \\
\hline 6 & & 5.38 & 134.8 \\
\hline 8 & & 3.84 & 85.93 \\
\hline 10 & & 1.78 & 40.68 \\
\hline
\end{tabular}

In the case of cultured transgenic roots morphology, two different morphological forms have been observed: highly ramified and compact structures. For cultures maintained at $\alpha \leq 6^{\circ}$ and $\omega \leq 20 \mathrm{~min}^{-1}$ the morphology was highly ramified, as for typical cultures performed in Erlenmeyer flask. But in other cultures, the hairy roots agglomerated into compact structures.

\section{Conclusion}

Morphology of transgenic roots strongly depended on conditions of wave-assisted agitation supported by the WAVE 25 bioreactor system. Hairy roots grew as typical highly ramified biomass at low sets of $\alpha$ and $\omega$. Intensified wave-assisted agitation resulted in the agglomeration of hairy roots into compact structures. The highest increase of $R$. graeca transgenic roots fresh biomass, i.e., over $800 \%$, has been observed for cultures performed at $\alpha=2^{\circ}$ and $\omega=20 \mathrm{~min}^{-1}$. In the case of secondary metabolites secretion, the highest level of naphthoquinone-analogues, i.e., $420.0 \mu \mathrm{g}$, has been observed for cultures performed at $\alpha=6^{0}$ and $\omega=10 \mathrm{~min}^{-1}$.

\section{Acknowledgements}

Research was funded by (POB Biotechnology and Biomedical Engineering) of Warsaw University of Technology within the Excellence Initiative: ResearchUniversity (IDUB) programme.

\section{References}

[1] Ochoa-Villarreal, M., Howat, S., Hong, S., Jang, M. O., Jin, Y.-W., Lee, E.-K., Loake, G. L. (2016). Plant cell cultures strategies for the production of natural products. BMB Reports, 49(3), 149-158. https://doi.org/10.5483/BMBRep.2016.49.3.264

[2] Nowak, B., Kawka, M., Wierzchowski, K., Sykłowska-Baranek, K., Pilarek, M. (2021). MTMS-Based Aerogel Constructs for Immobilization of Plant Hairy Roots: Effects on Proliferation of Rindera graeca Biomass and Extracellular Secretion of Naphthoquinones. Journal of Functional Biomaterials, 12, 19. https://doi.org/10.3390/jfb12010019

[3] Wierzchowski, K., Grabowska, I., Pilarek, M., (2020). Efficient propagation of suspended HL-60 cells in a disposable bioreactor supporting wave-induced agitation at various Reynolds number, Bioprocess and Biosystems Engineering, 43, 1973-1985. https://doi.org/10.1007/s00449-020-02386-6 